\title{
RADIAL SYMMETRY OF LARGE SOLUTIONS OF NONLINEAR ELLIPTIC EQUATIONS
}

\author{
STEVEN D. TALIAFERRO
}

(Communicated by Jeffrey Rauch)

Abstract. We give conditions under which all $C^{2}$ solutions of the problem

$$
\begin{aligned}
& \Delta u=f(|x|, u), \quad x \in \mathbb{R}^{n}, \\
& \lim _{|x| \rightarrow \infty} u(x)=\infty
\end{aligned}
$$

are radial. We assume $f(|x|, u)$ is positive when $|x|$ and $u$ are both large and positive. Since this problem with $f(|x|, u)=u$ has non-radial solutions, we rule out this possibility by requiring that $f(|x|, u)$ grow superlinearly in $u$ when $|x|$ and $u$ are both large and positive. However we make no assumptions on the rate of growth of solutions.

\section{INTRODUCTION}

In this paper we give conditions under which all $C^{2}$ solutions of the problem

$$
\begin{aligned}
& \Delta u=f(|x|, u), \quad x \in \mathbb{R}^{n}(n \geq 3), \\
& \lim _{|x| \rightarrow \infty} u(x)=\infty
\end{aligned}
$$

are radial. We assume $f(|x|, u)$ is positive when $|x|$ and $u$ are both large and positive. Since problem $(1 \mathrm{a}, \mathrm{b})$ with $f(|x|, u)=u$ has non-radial solutions (see Section 2), we rule out this possibility by requiring that $f(|x|, u)$ grow superlinearly in $u$ when $|x|$ and $u$ are both large and positive (see condition (f2) in Section 2). However we make no assumptions on the rate of growth of solutions.

In contrast to the above-mentioned necessity of a growth condition on $f$ in problem $(1 \mathrm{a}, \mathrm{b}), \mathrm{Li}$ and $\mathrm{Ni}[\mathrm{LN} 2]$ proved the following theorem concerning the wellstudied problem

$$
\begin{aligned}
& \Delta u=f(|x|, u), \quad x \in \mathbb{R}^{n}, \\
& \lim _{|x| \rightarrow \infty} u(x)=0
\end{aligned}
$$

which makes assumptions on neither the rate of growth of $f(|x|, u)$ in $u$ nor the rate of decay of solutions.

Received by the editors July 22, 1994; this paper was presented at the Special Session on Nonlinear Boundary Value Problems, 898th Meeting of the AMS, Hartford, CT, March 4, 1995. 1991 Mathematics Subject Classification. Primary 35J60. 
Theorem [LN2]. Suppose for $0 \leq r<\infty$ and $0 \leq s<\infty$ that $f(r, s)$ is continuous, Lipschitz in $s$, and non-decreasing (strictly increasing) in $r$. If $f_{s}(r, s) \geq 0$ for $r$ large and for $s$ small and positive, and $u(x)$ is a positive $C^{2}$ solution of $(2 \mathrm{a}, \mathrm{b})$, then $u(x)$ must be radial about some point (about the origin).

Our procedure for studying the radial symmetry of solutions of problem $(1 \mathrm{a}, \mathrm{b})$ has two parts. The first part, which contains most of the technical difficulties, consists of showing that the difference of any two solutions of problem $(1 \mathrm{a}, \mathrm{b})$ tends to zero as $|x| \rightarrow \infty$. (Note that this is trivially true for problem $(2 \mathrm{a}, \mathrm{b})$.) Here we use some results in $[\mathrm{T}]$ on the asymptotic behavior as $|x| \rightarrow \infty$ of solutions of $(1 \mathrm{a}, \mathrm{b})$. For the convenience of the reader, these results are stated in the appendix.

The second part consists of standard applications of the maximum principle and an adaptation to problem $(1 \mathrm{a}, \mathrm{b})$ of the Alexandrov-Serrin moving plane procedure as applied by $\mathrm{Li}$ and $\mathrm{Ni}[\mathrm{LN} 2]$ to problem $(2 \mathrm{a}, \mathrm{b})$.

When $n=2$ the problem analogous to problem $(1 \mathrm{a}, \mathrm{b})$ is

$$
\begin{aligned}
& \Delta u=f(|x|, u), \quad x \in \mathbb{R}^{2}, \\
& \lim _{|x| \rightarrow \infty} \frac{u(x)}{\log |x|}=\infty .
\end{aligned}
$$

Theorem 1 below holds for this two-dimensional problem provided the expression $r^{2 n-2} g(r, s)$ in condition (f3) below is replaced with $r^{2}(\log r)^{3} g(r, s \log r)$.

Radial properties of solutions on $\mathbb{R}^{n}$ of non-linear elliptic equations have also been studied in [CL, GNN, LN1, Z1, Z2].

\section{Statement of Results}

To state our results for problem $(1 \mathrm{a}, \mathrm{b})$, we introduce the following assumptions on $f(r, s)$ :

(f1) $f$ and $f_{s}$ are continuous on $[0, \infty) \times \mathbb{R}$;

(f2) there exist $\lambda>1, r_{0}>0$, and $s_{0}>0$ such that

$$
f(r, v s) \geq v^{\lambda} f(r, s)>0
$$

for $v \geq 1, r \geq r_{0}$, and $s \geq s_{0}$;

(f3) $\frac{f(r, s)}{g(r, s)} \rightarrow 1$ as $(r, s) \rightarrow(\infty, \infty)$ for some function $g(r, s)$ with the property that it and its partial derivative $g_{s}(r, s)$ are defined, continuous, and positive for $r \geq r_{0}$ and $s \geq s_{0}$ and with the property that $r^{2 n-2} g(r, s)$ is monotone ${ }^{1}$ in $r$ on $\left[r_{0}, \infty\right) \times\left[s_{0}, \infty\right)$.

Our main result is

Theorem 1. Suppose that $f(r, s)$ satisfies conditions (f1-3) and that $u(x)$ is a $C^{2}$ solution of problem $(1 \mathrm{a}, \mathrm{b})$. Let $s_{m}=\min _{x \in \mathbb{R}^{n}} u(x)$.

(i) If $f_{s}$ is non-negative on $[0, \infty) \times\left[s_{m}, \infty\right)$, then $u(x)$ is radial about the origin.

(ii) If $f(r, s)$ is monotone (strictly monotone) in $r$ on $[0, \infty) \times\left[s_{m}, \infty\right)$, then $u(x)$ is radial about some point (about the origin).

\footnotetext{
${ }^{1}$ We say a function $g(r, s)$ is monotone in $r$ on $\left[r_{0}, \infty\right) \times\left[s_{0}, \infty\right)$ if either (i) for each $s \in\left[s_{0}, \infty\right)$, $g(r, s)$ is non-decreasing in $r$ on $\left[r_{0}, \infty\right)$, or (ii) for each $s \in\left[s_{0}, \infty\right), g(r, s)$ is non-increasing in $r$ on $\left[r_{0}, \infty\right)$.
} 
If $\alpha_{1}, \ldots, \alpha_{n}$ are positive constants such that $\alpha_{1}^{2}+\cdots+\alpha_{n}^{2}=1$, then a non-radial solution of

$$
\Delta u=u, \quad \lim _{|x| \rightarrow \infty} u(x)=\infty
$$

is $u\left(x_{1}, \ldots, x_{n}\right)=\left(\cosh \alpha_{1} x_{1}\right) \cdots\left(\cosh \alpha_{n} x_{n}\right)$. Thus condition (f2) in Theorem 1 cannot be weakened by allowing $\lambda=1$.

By dividing the displayed inequalities in condition (f2) by $(v s)^{\lambda}$ we find that condition (f2) is equivalent to the following condition:

$(\mathfrak{f} 2)^{\prime}$ there exist $\lambda>1, r_{0}>0$, and $s_{0}>0$ such that the expression $s^{-\lambda} f(r, s)$ is positive and non-decreasing in $s$ on $\left[r_{0}, \infty\right) \times\left[s_{0}, \infty\right)$.

Theorem 2. Suppose that $f$ satisfies conditions (f1-3). If the problem

$$
\begin{aligned}
& \Delta u=f(|x|, u), \quad x \in \mathbb{R}^{n}(n \geq 3) \text { and }|x| \text { large }, \\
& \lim _{|x| \rightarrow \infty} u(x)=\infty
\end{aligned}
$$

has a $C^{2}$ solution, then

$$
\int_{r_{0}}^{\infty} r f(r, s) d r<\infty, \quad \text { for all } \quad s \geq s_{0}
$$

and problem $(3 \mathrm{a}, \mathrm{b})$ has a $C^{2}$ radial solution $u_{0}(|x|)$, which is strictly increasing in $|x|$, such that all $C^{2}$ solutions $u(x)$ of $(3 \mathrm{a}, \mathrm{b})$ satisfy

$$
\lim _{|x| \rightarrow \infty}\left[u(x)-u_{0}(|x|)\right]=0 .
$$

\section{ProOfs}

In this section we prove Theorems 1 and 2. First we show how Theorem 1 follows from Theorem 2 and then we prove Theorem 2.

Proof of part (i) of Theorem 1 . Let $T$ be a unitary operator on $\mathbb{R}^{n}$ and let $u_{T}(x)=$ $u(T x)$. Since $\Delta\left(u_{T}\right)=(\Delta u)_{T}$, we find that $u_{T}$ is also a solution of $(1 \mathrm{a}, \mathrm{b})$. Thus

$$
\Delta\left(u_{T}-u\right)=f\left(|x|, u_{T}\right)-f(|x|, u)
$$

and by Theorem 2 ,

$$
\lim _{|x| \rightarrow \infty}\left(u_{T}-u\right)(x)=0
$$

Since $f_{s}(r, s) \geq 0$ on $[0, \infty) \times\left[s_{m}, \infty\right)$, it follows from $(5 \mathrm{a}, \mathrm{b})$ and the maximum principle applied to $u_{T}-u$ that $u_{T}-u \equiv 0$ on $\mathbb{R}^{n}$. Since $T$ is an arbitrary unitary operator, $u$ is radial about the origin.

Proof of part (ii) of Theorem 1. With the aid of Theorem 2, part (ii) of Theorem 1 can be proved by using the Alexandrov-Serrin moving plane procedure similar to the way it was used by $\mathrm{Li}$ and $\mathrm{Ni}[\mathrm{LN} 2]$. We can show the essential differences between the procedure for our problem and the procedure for the problem in [LN2] by starting the procedure near $\infty$. The remainder of the procedure will not be given 
because it only involves making similar changes to the remainder of the procedure in [LN2].

For each $\lambda \in \mathbb{R}$, denote the reflection of a point $x=\left(x_{1}, \ldots, x_{n}\right) \in \mathbb{R}^{n}$ about the hyperplane $T_{\lambda}=\left\{x \in \mathbb{R}^{n}: x_{1}=\lambda\right\}$ by $x^{\lambda}$. Then $x^{\lambda}=\left(2 \lambda-x_{1}, x_{2}, \ldots, x_{n}\right)$. Let $\Sigma_{\lambda}=\left\{x \in \mathbb{R}^{n}: x_{1}<\lambda\right\}$ and

$$
\Lambda=\left\{\lambda \in \mathbb{R}: u(x)<u\left(x^{\lambda}\right) \text { for all } x \in \Sigma_{\lambda} \text { and } \frac{\partial u}{\partial x_{1}}>0 \text { on } T_{\lambda}\right\} .
$$

We now start the moving plane procedure near $\infty$; that is, we show $\lambda \in \Lambda$ for $\lambda$ large and positive. By (f1) and (f2) there exists $R_{0}>0$ such that

$$
f_{s}(r, s) \geq 0 \quad \text { for } \quad r \geq R_{0} \quad \text { and } \quad s \geq R_{0} .
$$

Choose $R_{2}>R_{1}>R_{0}$ such that

$$
\min _{|x| \geq R_{1}} u(x)>R_{0} \quad \text { and } \quad \min _{|x| \geq R_{2}} u(x)>\max _{|x| \leq R_{1}} u(x) .
$$

Choose $\lambda \geq R_{2}$ and let $w_{\lambda}(x)=u(x)-u\left(x^{\lambda}\right)$ for $x \in \Sigma_{\lambda}$. Let $u_{0}(|x|)$ be as in Theorem 2. Since

$$
\left|x^{\lambda}\right|>|x| \quad \text { for } \quad x \in \Sigma_{\lambda},
$$

we find by Theorem 2 that

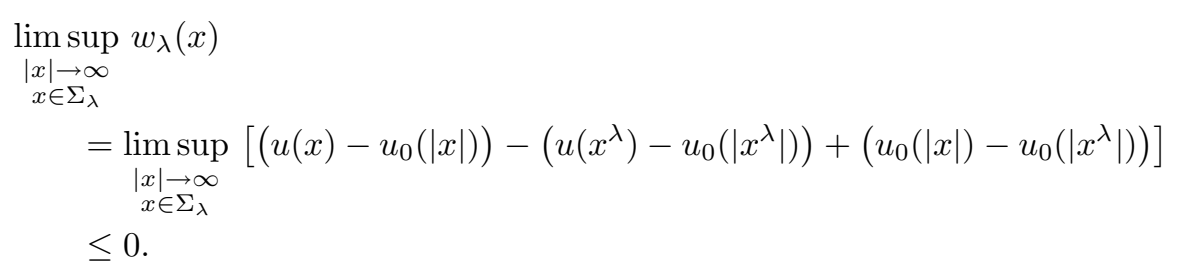

Since $f(r, s)$ is monotone in $r$ on $[0, \infty) \times\left[s_{m}, \infty\right)$ and (4) holds, we see that $f(r, s)$ is non-increasing in $r$ on $[0, \infty) \times\left[s_{m}, \infty\right)$. Thus by (8),

$$
\begin{aligned}
\Delta w_{\lambda}(x) & \geq f(|x|, u(x))-f\left(|x|, u\left(x^{\lambda}\right)\right) \\
& =c(x) w_{\lambda}(x), \quad x \in \Sigma_{\lambda},
\end{aligned}
$$

where $c(x)=\int_{0}^{1} f_{s}\left(|x|, u\left(x^{\lambda}\right)+t\left(u(x)-u\left(x^{\lambda}\right)\right)\right) d t$. It follows from (6), (7), and (8) that

$$
c(x) \geq 0 \quad \text { for } \quad x \in \Sigma_{\lambda}-B_{R_{1}}(0)
$$

and

$$
w_{\lambda}(x)<0 \quad \text { for } \quad|x| \leq R_{1} .
$$

Therefore $w_{\lambda}$ satisfies

$$
\begin{array}{cc}
\Delta w_{\lambda}-c(x) w_{\lambda} \geq 0 & \text { on } \quad \Sigma_{\lambda}-\overline{B_{R_{1}}(0)}, \\
w_{\lambda} \leq 0 & \text { on } \quad \partial\left(\Sigma_{\lambda}-\overline{B_{R_{1}}(0)}\right),
\end{array}
$$

$$
\limsup \underset{x \in \Sigma_{\lambda}-\overline{B_{R_{1}}(0)}}{|x| \rightarrow \infty} w_{\lambda}(x) \leq 0 .
$$


Thus it follows from (9), (10), the strong maximum principle, and the Hopf boundary lemma that $w_{\lambda}(x)<0$ in $\Sigma_{\lambda}-\overline{B_{R_{1}}(0)}$ and $\frac{\partial w_{\lambda}}{\partial x_{1}}>0$ on $T_{\lambda}$. Hence by (10), $\lambda \in \Lambda$; that is $\left[R_{2}, \infty\right) \subset \Lambda$. The moving plane procedure is now completed similar to the way it was completed in [LN2].

Proof of Theorem 2. Let $g$ be as in condition (f3). Then $g$ satisfies conditions (g1) and (g3) in the appendix. Let $\lambda$ be as in (f2) and $d>1$. Then by (f2) and (f3)

$$
\liminf _{(r, s) \rightarrow(\infty, \infty)} \inf _{v>d} \frac{g(r, v s)}{v^{(1+\lambda) / 2} g(r, s)} \geq d^{(\lambda-1) / 2}>1 .
$$

Hence $g$ also satisfies (g2). So by (f3), $f$ and $g$ satisfy the hypotheses of Lemma A.1 in the appendix. Since, by assumption, problem (3a,b) has a $C^{2}$ solution, it follows from part (ii) of Lemma A.1 that problem $(20 \mathrm{a}, \mathrm{b})$ has a $C^{2}$ radial solution $y(|x|)$. Thus by part (iii) of Lemma A.1 and (f3), we have that (4) holds, and by part (i) of Lemma A.1, the ratio of any two solutions of $(3 \mathrm{a}, \mathrm{b})$ tends to one as $|x| \rightarrow \infty$. Hence, since under the change of variables

$$
z(t)=u(|x|), \quad t=-|x|^{2-n},
$$

the problem of finding radial solutions $u(|x|)$ of problem $(3 \mathrm{a}, \mathrm{b})$ transforms into the problem of finding solutions $z(t)$ of

$$
\begin{aligned}
& z^{\prime \prime}=F(t, z), \quad t \text { negative and small, } \\
& \lim _{t \rightarrow 0^{-}} z(t)=\infty
\end{aligned}
$$

where $f(r, s)=(n-2)^{2} r^{2-2 n} F\left(-r^{2-n}, s\right)$, it follows that the ratio of any two solutions of problem $(12 \mathrm{a}, \mathrm{b})$ tends to one as $t \rightarrow 0^{-}$.

From this last conclusion we show through each point $\left(t_{1}, s_{1}\right) \in\left[t_{0}, 0\right) \times\left[s_{0}, \infty\right)$, where $t_{0}=-r_{0}^{2-n}$, that there can be at most one solution of $(12 \mathrm{a}, \mathrm{b})$ whose graph is contained in $\left[t_{1}, 0\right) \times\left[s_{0}, \infty\right)$. Suppose to the contrary that $z_{1}(t) \not \equiv z_{2}(t)$ are two such solutions. Then, since $f$ is $C^{1}$ in $s, z_{1}^{\prime}\left(t_{1}\right) \neq z_{2}^{\prime}\left(t_{1}\right)$ and we can assume $z_{1}^{\prime}\left(t_{1}\right)>z_{2}^{\prime}\left(t_{1}\right)$. Let

$$
\zeta=\zeta(t)=-\int_{t}^{0} \frac{d \tau}{z_{2}(\tau)} \quad \text { and } \quad v(\zeta)=\frac{z_{1}(t)}{z_{2}(t)}
$$

Let $\zeta_{1}=\zeta\left(t_{1}\right)$. Then $v\left(\zeta_{1}\right)=1$,

$$
v^{\prime}\left(\zeta_{1}\right)=s_{1}\left[z_{1}^{\prime}\left(t_{1}\right)-z_{2}^{\prime}\left(t_{1}\right)\right]>0
$$

and by (f2),

$$
\begin{aligned}
v^{\prime \prime}(\zeta) & =v(\zeta) z_{2}(t)^{3} F\left(t, z_{2}(t)\right)\left[\frac{F\left(t, v(\zeta) z_{2}(t)\right)}{v(\zeta) F\left(t, z_{2}(t)\right)}-1\right] \\
& \geq 0 \quad \text { for } \quad v(\zeta) \geq 1
\end{aligned}
$$

Hence $v^{\prime \prime}(\zeta) \geq 0$ for $\zeta_{1} \leq \zeta<0$ and $\liminf _{\zeta \rightarrow 0^{-}} v(\zeta)>1$, which contradicts the fact that the ratio of any two solutions of problem $(12 \mathrm{a}, \mathrm{b})$ tends to one as $t \rightarrow 0^{-}$. 
Next we show that the difference of any two solutions of problem $(12 \mathrm{a}, \mathrm{b})$ tends to zero as $t \rightarrow 0^{-}$. Let $z_{1}(t) \not \equiv z_{2}(t)$ be two solutions of $(12 \mathrm{a}, \mathrm{b})$. Clearly there exists $t_{1} \in\left[t_{0}, 0\right)$ such that for $t_{1} \leq t<0$ we have $z_{1}(t)>s_{0}, z_{2}(t)>s_{0}, z_{1}^{\prime}(t)>0$, and $z_{2}^{\prime}(t)>0$. By the previous paragraph, $z_{1}(t) \neq z_{2}(t)$ for $t_{1} \leq t<0$. Hence we can assume that $z_{1}(t)>z_{2}(t)$ for $t_{1} \leq t<0$. Suppose $z_{1}^{\prime}\left(t_{2}\right)>z_{2}^{\prime}\left(t_{2}\right)$ for some $t_{2} \in\left[t_{1}, 0\right)$. Let $z_{3}(t)$ be the solution of $(12 \mathrm{a}, \mathrm{b})$ satisfying $z_{3}\left(t_{2}\right)=z_{2}\left(t_{2}\right)$ and $z_{3}^{\prime}\left(t_{2}\right)=z_{1}^{\prime}\left(t_{2}\right)$. Since by (f2), $f(r, s)$ is non-decreasing in $s$, it follows from a simple comparison argument that $z_{2}(t)<z_{3}(t)<z_{1}(t)$ for $t \in\left(t_{2}, 0\right)$. Thus $z_{3}(t)$ is a solution of $(12 \mathrm{a}, \mathrm{b})$ which contradicts the previous paragraph. Therefore $z_{1}(t)-z_{2}(t)$ is positive and non-increasing on the interval $\left[t_{1}, 0\right)$.

Let $a=\lim _{t \rightarrow 0^{-}} z_{1}(t)-z_{2}(t)$. Suppose $a>0$. By (f2) we find that

$$
\begin{aligned}
z_{1}^{\prime \prime}(t)-z_{2}^{\prime \prime}(t) & \geq F\left(t, z_{2}(t)+a\right)-F\left(t, z_{2}(t)\right) \\
& \geq\left[\left(\frac{z_{2}(t)+a}{z_{2}(t)}\right)^{\lambda}-1\right] F\left(t, z_{2}(t)\right) \\
& \geq \frac{\lambda a}{z_{2}(t)} F\left(t, z_{2}(t)\right) \quad \text { for } \quad t_{1} \leq t<0 .
\end{aligned}
$$

Integrating (13) from $t$ to 0 we get

$$
-\left[z_{1}^{\prime}(t)-z_{2}^{\prime}(t)\right] \geq \lambda a \int_{t}^{0} \frac{F\left(\tau, z_{2}(\tau)\right)}{z_{2}(\tau)} d \tau \quad \text { for } \quad t_{1} \leq t<0 .
$$

Integrating (14) from $t_{1}$ to 0 and interchanging the order of integration gives

$$
\begin{aligned}
{\left[z_{1}\left(t_{1}\right)-z_{2}\left(t_{1}\right)\right]-a } & \geq \lambda a \int_{t_{1}}^{0}\left(\tau-t_{1}\right) \frac{F\left(\tau, z_{2}(\tau)\right)}{z_{2}(\tau)} d \tau \\
& \geq \frac{\lambda a\left|t_{1}\right|}{2} \int_{t_{1} / 2}^{0} \frac{F\left(\tau, z_{2}(\tau)\right)}{z_{2}(\tau)} d \tau
\end{aligned}
$$

Hence

$$
\int_{t_{1}}^{0} \frac{F\left(\tau, z_{2}(\tau)\right)}{z_{2}(\tau)} d \tau<\infty .
$$

But integrating $z_{2}^{\prime \prime}(t)=F\left(t, z_{2}(t)\right)$ twice from $t_{1}$ to $t$ gives

$$
\left|z_{2}(t)\right| \leq c+\left|t_{1}\right| \int_{t_{1}}^{t} \frac{F\left(\tau, z_{2}(\tau)\right)}{z_{2}(\tau)} z_{2}(\tau) d \tau
$$

where $c=z_{2}\left(t_{1}\right)+\left|t_{1}\right| z_{2}^{\prime}\left(t_{1}\right)$. Therefore by Gronwall's inequality and (15) we have

$$
\left|z_{2}(t)\right| \leq c \exp \left(\left|t_{1}\right| \int_{t_{1}}^{0} \frac{F\left(\tau, z_{2}(\tau)\right)}{z_{2}(\tau)} d \tau\right)<\infty
$$

for $t_{1} \leq t<0-$ a contradiction. Thus $a=0$ and we have therefore shown that the difference of any two solutions of problem $(12 \mathrm{a}, \mathrm{b})$ tends to zero as $t \rightarrow 0^{-}$. Hence, 
since under the change of variables (11) the problem of finding solutions $z(t)$ of problem $(12 \mathrm{a}, \mathrm{b})$ transforms into the problem of finding radial solutions $u(|x|)$ of problem $(3 \mathrm{a}, \mathrm{b})$, it follows that the difference of any two solutions of problem $(3 \mathrm{a}, \mathrm{b})$ which are radial about the origin tends to zero as $|x| \rightarrow \infty$. Also, since any solution $z(t)$ of $(12 \mathrm{a}, \mathrm{b})$ is clearly strictly increasing for $t$ negative and near zero, it follows that any radial solution $u(|x|)$ of $(3 \mathrm{a}, \mathrm{b})$ is strictly increasing in $|x|$ for $|x|$ large.

Let $u(x)$ be a solution of $(3 \mathrm{a}, \mathrm{b})$. Then, as shown above, (4) holds. Since $f$ satisfies conditions (f1) and (f2), it follows that $f$ satisfies the conditions imposed on $g$ in conditions (g1) and (g2) in the appendix. So $f$ satisfies the hypotheses of Lemma A.2 in the appendix. Let $s_{1}>s_{0}$. Let $r_{1}>r_{0}$ be as in Lemma A.2. Since $u(x)$ satisfies (3b), there exists $r_{2}>r_{1}$ such that $u(x)>s_{1}+1$ for $|x| \geq r_{2}$. Choose $s_{2} \in\left(s_{1}+1, \min _{|x|=r_{2}} u(x)\right)$. Let $u_{0}(r), u_{1}(r), \ldots$ be as in Lemma A.2. Then, for $k \geq 1$, we have $u(x)-u_{k}(|x|)>0$ for $|x|=r_{2}, \lim _{|x| \rightarrow \infty} u(x)-u_{k}(|x|)=\infty$ and

$$
\begin{aligned}
\Delta\left(u(x)-u_{k}(|x|)\right) & =f(|x|, u(x))-f\left(|x|, u_{k}(|x|)\right) \\
& <0 \quad \text { for } \quad u(x)<u_{k}(|x|)
\end{aligned}
$$

because, by (f2), $f(r, s)$ is strictly increasing in $s$ on $\left[r_{0}, \infty\right) \times\left[s_{0}, \infty\right)$. It therefore follows from the maximum principle that $u(x) \geq u_{k}(|x|)$ for $|x| \geq r_{2}$ and letting $k \rightarrow \infty$ we find that

$$
u(x) \geq u_{0}(|x|) \quad \text { for } \quad|x| \geq r_{2} .
$$

Now choose $s_{2} \in\left(\max _{|x|=r_{2}} u(x), \infty\right)$ and let $U_{0}(r), U_{1}(r), \ldots$ be as in Lemma A.2. Then, for $k \geq 1$, we have $u(x)-U_{k}(|x|)<0$ for $|x|=r_{2}, \lim _{|x| \rightarrow \rho_{k}^{-}} u(x)-U_{k}(|x|)=-\infty$ and $\Delta\left(u(x)-U_{k}(|x|)\right)>0$ for $u(x)>U_{k}(|x|)$. Therefore, by the maximum principle, $u(x) \leq U_{k}(|x|)$ for $r_{2} \leq|x|<\rho_{k}$ and letting $k \rightarrow \infty$ we see that

$$
u(x) \leq U_{0}(|x|) \quad \text { for } \quad|x| \geq r_{2} .
$$

Since, as shown above, the difference of any two solutions of problem (3a,b) which are radial about the origin tends to zero as $|x| \rightarrow \infty$, Theorem 2 follows from (16) and (17).

\section{APPENDIX}

In $[\mathrm{T}]$ we studied the radial properties and asymptotic behavior of solutions of the problem

$$
\begin{aligned}
& \Delta u=f(|x|, u), \quad x \in \mathbb{R}^{n}(n \geq 3) \text { and }|x| \text { large, } \\
& \lim _{|x| \rightarrow \infty} u(x)=\infty
\end{aligned}
$$

Some of our results required that

$$
\frac{f(r, s)}{g(r, s)} \rightarrow 1 \quad \text { as } \quad(r, s) \rightarrow(\infty, \infty)
$$


for some function $g(r, s)$ such that

(g1) for some $r_{0}>0$ and $s_{0}>0, g(r, s)$ and $g_{s}(r, s)$ are defined, continuous, and positive for $r \geq r_{0}$ and $s \geq s_{0}$;

(g2) there exists $\gamma>1$ such that for all $d>1$

$$
\liminf _{(r, s) \rightarrow(\infty, \infty)} \inf _{v>d} \frac{g(r, v s)}{v^{\gamma} g(r, s)}>1
$$

(g3) $r^{2 n-2} g(r, s)$ is monotone in $r$ on $\left[r_{0}, \infty\right) \times\left[s_{0}, \infty\right)$.

In $[\mathrm{T}]$ we proved

Lemma A.1. Suppose $g$ satisfies (g1-3), $f(r, s)$ is continuous on $\left[r_{0}, \infty\right) \times\left[s_{0}, \infty\right)$, and (19) holds.

(i) If $u(x)$ is a $C^{2}$ solution of $(18 \mathrm{a}, \mathrm{b})$ then for each $C^{2}$ radial solution $y(|x|)$ of

$$
\begin{aligned}
& \Delta y=g(|x|, y), \quad x \in \mathbb{R}^{n} \text { and }|x| \text { large }, \\
& \lim _{|x| \rightarrow \infty} y(x)=\infty
\end{aligned}
$$

$u(x)$ satisfies

$$
\lim _{|x| \rightarrow \infty} \frac{u(x)}{y(|x|)}=1 .
$$

(ii) If problem $(20 \mathrm{a}, \mathrm{b})$ has no $C^{2}$ radial solution $y(|x|)$, then problem $(18 a, b)$ has no $C^{2}$ solution.

(iii) Problem $(20 \mathrm{a}, \mathrm{b})$ has a $C^{2}$ radial solution $y(|x|)$ if and only if

$$
\int_{r_{0}}^{\infty} r g(r, s) d r<\infty
$$

for all $s \geq s_{0}$.

Lemma A.2. Suppose that $f(r, s)$ satisfies the hypotheses imposed on $g(r, s)$ in (g1-2) and that

$$
\int_{r_{0}}^{\infty} r f(r, s) d r<\infty
$$

for all $s \geq s_{0}$. Then for each $s_{1}>s_{0}$ there exists $r_{1}>r_{0}$ such that if $s_{2} \geq s_{1}+1$ and $r_{2} \geq r_{1}$, then there exist a sequence $\left\{\rho_{k}\right\}_{k=1}^{\infty}$ in $\left(r_{2}, \infty\right)$ satisfying $\lim _{k \rightarrow \infty} \rho_{k}=\infty$ and $C^{2}$ functions $u_{0}(r), u_{1}(r), \ldots$ and $U_{0}(r), U_{1}(r), \ldots$ whose ranges are contained in $\left(s_{1}, \infty\right)$ such that

(i) $U_{0}(|x|)$ and $u_{0}(|x|), u_{1}(|x|), \ldots$ are solutions of

$$
\begin{aligned}
& \Delta u=f(|x|, u), \quad|x| \geq r_{2}, \\
& u(x)=s_{2}, \quad|x|=r_{2} ;
\end{aligned}
$$

(ii) for each positive integer $k, U_{k}(|x|)$ is a solution of

$$
\begin{aligned}
& \Delta u=f(|x|, u), \quad r_{2} \leq|x|<\rho_{k}, \\
& u(x)=s_{2}, \quad|x|=r_{2}, \\
& \lim _{|x| \rightarrow \rho_{k}^{-}} u(x)=\infty ;
\end{aligned}
$$


(iii) $\lim _{r \rightarrow \infty} u_{0}(r)=\lim _{r \rightarrow \infty} U_{0}(r)=\infty$;

(iv) for each positive integer $k, u_{k}(r)$ has a finite limit as $r \rightarrow \infty$;

(v) for each $r \geq r_{2}$, we have

$$
\lim _{k \rightarrow \infty} u_{k}(r)=u_{0}(r) \quad \text { and } \quad \lim _{k \rightarrow \infty} U_{k}(r)=U_{0}(r)
$$

\section{REFERENCES}

[CL] W. Chen and C. Li, Classification of solutions to a singular nonlinear elliptic equation, preprint.

[GNN] B. Gidas, W-M Ni, and L. Nirenberg, Symmetry of positive solutions of nonlinear elliptic equations in $\mathbb{R}^{n}$, in Mathematical Analysis and Applications (L. Nachbin, ed.), Academic Press, 1981, pp. 369-402. MR 84a:35083

[LN1] Y. Li and W-M Ni, On the asymptotic behavior and radial symmetry of positive solutions of semilinear elliptic equations in $\mathbb{R}^{n}$, I. Asymptotic behavior, II Radial symmetry, Arch. Rat. Mech. Anal. 118 (1992), 195-244. MR 93e:35036

[LN2] Y. Li and W-M Ni, Radial symmetry of positive solutions of nonlinear elliptic equations in $\mathbb{R}^{n}$, Commun. Part. Diff. Eq. 18 (1993), 1043-1054. MR 95c:35026

[T] S.D. Taliaferro, Are solutions of almost radial nonlinear elliptic equations almost radial?, Commun. Part. Diff. Eq., (in press)

[Z1] H. Zou, A local harnack inequality and classification of positive solutions of $\Delta u+u^{p}=0$ in $\mathbb{R}^{n}$, preprint.

[Z2] H. Zou, Symmetry and local behavior of positive solutions of $\Delta u+u^{p}=0$ in $\mathbb{R}^{n}$, preprint.

Mathematics Department, Texas A\&M University, College Station, Texas 77843

E-mail address: stalia@math.tamu.edu 